\title{
LA DÉMOCRATIE SELON PLATON OU DE L'IGNORANCE COLLECTIVE ÉRIGÉE EN PRINCIPE POLITIQUE
}

\author{
Marc-Antoine Gavray
}

Vrin | «Cahiers philosophiques »

$2020 / 1 \mathrm{~N}^{\circ} 160$ | pages 23 à 38

ISSN 0241-2799

ISBN 9782711660124

Article disponible en ligne à l'adresse :

https://www.cairn.info/revue-cahiers-philosophiques-2020-1-page-23.htm

Distribution électronique Cairn.info pour Vrin.

(C) Vrin. Tous droits réservés pour tous pays.

La reproduction ou représentation de cet article, notamment par photocopie, n'est autorisée que dans les limites des conditions générales d'utilisation du site ou, le cas échéant, des conditions générales de la licence souscrite par votre établissement. Toute autre reproduction ou représentation, en tout ou partie, sous quelque forme et de quelque manière que ce soit, est interdite sauf accord préalable et écrit de l'éditeur, en dehors des cas prévus par la législation en vigueur en France. Il est précisé que son stockage dans une base de données est également interdit. 


\section{Embarras de la démocratie}

\section{LA DÉMOCRATIE SELON PLATON OU DE L'IGNORANCE COLLECTIVE ÉRIGÉE EN PRINCIPE POLITIQUE'}

Marc-Antoine Gavray

La critique platonicienne de la démocratie est résolument philosophique, contestant le modèle anthropologique et épistémologique sous-jacent au nom des aberrations historiques auxquelles il est condamné. Cet article examine les deux principaux moteurs de l'analyse platonicienne : 1) l'absence d'unité, qui se traduit par une oligarchie de fait et par une dispersion des citoyens, plus soucieux de leur liberté individuelle que de l'intérêt de la cité ; 2) une théorie de la connaissance qui, aussi implicite soit-elle, dissocie savoir et pouvoir. Il en résulte une prise de distance à l'égard d'un modèle où I'homme démocratique serait jugé compétent par nature à exercer des responsabilités.

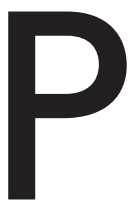

laton n'est pas vraiment un partisan de la démocratie. Faut-il l'en blâmer? C'est en effet la démocratie qui, à ses yeux, a condamné Socrate à la peine capitale. C'est elle également qui porte la responsabilité de la guerre interminable contre Sparte, sombre décor de sa jeunesse ${ }^{2}$. Sans même évoquer les manipulations des démagogues, la culture des tribunaux ni les débats interminables (ou mal terminés) qui l'animent, les raisons ne manquent pas de la critiquer. Or, si Platon la prend régulièrement pour cible, ce n'est pas au nom de considérations historiques et politiques de ce type. Son principal grief est philosophique : de son point de vue, c'est le modèle sur lequel la démocratie repose - la conception de l'homme et du savoir sous-jacent - qui est inadéquat. Les problèmes factuels, historiques, ne sont que les symptômes

1. Je remercie Stéphane Marchand non seulement de m'avoir encouragé à écrire ce texte, mais également de m'avoir fait profiter de ses remarques. Je n'en porte pas moins la responsabilité de son contenu.

2. La guerre du Péloponnèse débute en 431 et se termine en 404 . Platon naît quant à lui en 427 et passe donc son premier quart de siècle dans un climat de guerre continue, suivie par une période de répression violente puis de restauration de la démocratie qui s'achèvera notamment par la condamnation de Socrate. 
de postulats théoriques (implicites) qu'il identifie au gré des analyses. Platon critique la démocratie parce qu'elle ne peut rien produire d'autre que cela.

Le jugement paraîtra rétrograde, conservateur, voire inspiré par le pire des totalitarismes. Il suffit cependant de regarder les attaques - plus vives encore - que Platon formule à l'encontre de l'oligarchie ou de la tyrannie pour constater qu'il ne nourrit aucune haine particulière pour la démocratie. Sa critique relève plutôt d'une analyse sans compromis des défauts inhérents à ces régimes, qui aboutit à une hiérarchie dans le pire où la démocratie n'occupe pas la dernière marche ${ }^{3}$. Je voudrais en examiner les éléments concernant cette dernière afin d'en évaluer la pertinence et la vigueur. Mon but ne sera pas de donner raison à Platon ni même de le justifier, mais de remonter au principe de sa démarche, dans l'idée que son embarras vis-à-vis de la démocratie ne disparaît pas dans notre conception moderne : non pas Platon comme vestige historique, pas davantage comme modèle, mais comme un moteur de questionnement dans notre rapport à la démocratie et à la politique en général.

\section{Quelle unité pour la cité démocratique?}

Je prendrai pour fil conducteur deux éloges que Platon insère dans ses dialogues, tous deux placés dans la bouche d'une figure éminente de la démocratie. Cœur du Ménéxène, le premier est attribué à Aspasie, la femme du stratège Périclès, ce héros démocrate de la seconde moitié du ve siècle. Le second provient du Protagoras, où il est prononcé par le sophiste d'Abdère. Ces deux portraits idéalisés présentent la démocratie sous son meilleur jour, celui-là même que Platon interroge.

\section{Une aristocratie de fait}

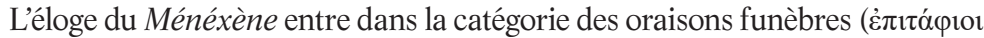
$\lambda$ ófor) - un genre épidictique consistant à louer les morts (au combat) et, à travers eux, la cité dont ils ont illustré les valeurs. La critique de Platon ne visera pas le principe de la mise en scène, le fait que la démocratie enjolive ses origines et son présent. Tout régime éprouve en effet le besoin de se raconter pour asseoir sa légitimité et persuader ses citoyens de la solennité des valeurs à respecter. Même les cités vertueuses de la République et des Lois n'échappent pas à la règle, se forgeant un discours modèle - un mensonge d'État - qui assure leur unité ${ }^{4}$. En ce sens, la démocratie recourt à la fiction pour remplir la fonction de transmission des valeurs, d'autant qu'aucun pouvoir fort n'y garantit la cohésion sociale ${ }^{5}$.

3. Pour une analyse des emprunts à la démocratie que Platon s'autorise dans une certaine mesure, lire l'article

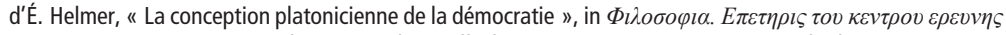
$\tau \eta \varsigma \varepsilon \lambda \lambda \eta v \imath \kappa \eta \varsigma \varphi \imath \lambda o \sigma o \varphi l \alpha \varsigma$, Athènes, Académie d'Athènes, 2010, p. 251-267. Cet article dresse en outre un bilan des tendances interprétatives du rapport de Platon à la démocratie que le lecteur pourra consulter (p. 251-252). Le livre récent d'Anders Dahl Sørensen (Plato on Democracy and Political Technē, Leiden, Brill, 2017) donne une vision plus large du dialogue de Platon avec la démocratie.

4. Cf. République, III, 414b-415d. Sur le «noble mensonge » et sa fonction dans la République, voir M. Schofield, Plato. Political Philosophy, Oxford, Oxford University Press, 2006, p. 284-309. Dans les Lois, c'est la fonction essentielle que remplissent les préambules, par exemple en IV, 715e-720e, où l'Athénien s'en justifie.

5. II suffit de voir les effets que Socrate, non sans une ironie décelée par Ménéxène, avoue éprouver à l'écoute de ce discours : il se sent comme envouté et grandi par le prestige des morts (Ménéxène, 235a-c). 
Comme l'a relevé Gregory Vlastos, la dimension parodique du texte est indéniable ${ }^{6}$. Platon ne prétend d'ailleurs pas faire œuvre historique, mais les mots qu'il prête à Aspasie traduisent sa perception du discours glorificateur de la démocratie. Examinons tout d'abord comment ce dernier met en évidence deux spécificités d'Athènes et du régime démocratique : l'excellence et l'homogénéité.

Car c'était alors le même régime ( $\pi$ o $\lambda \imath \tau \varepsilon i ́ \alpha)$ que de nos jours, le gouvernement

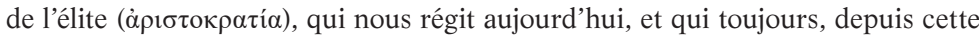
époque lointaine, s'est maintenu la plupart du temps. Celui-ci l'appelle démocratie

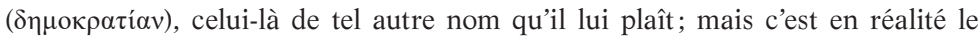

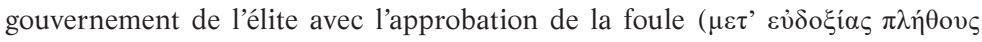
àpıбтократí; Ménéxène, 238c5-d2, trad. fr. L. Méridier) ${ }^{7}$.

Dans un contexte où la démocratie n'est qu'un régime parmi d'autres dont la supériorité est loin d'être l'évidence qui nous apparaît aujourd'hui, la stratégie de légitimation mise en œuvre par Aspasie consiste à affirmer que, si ce régime surpasse les autres, c'est parce qu'il revêt en réalité les traits d'une aristocratie, un régime où les meilleurs exercent le pouvoir : sa valeur générale transparaîtra de celle de ses gouvernants. Or, puisqu’en démocratie le pouvoir appartient au peuple dans son ensemble et sans distinction (à l'exclusion notable des femmes et des esclaves), il s'agit de poser que l'extension démocratique du pouvoir ne nuit en rien à la valeur des gouvernants ${ }^{8}$. Cela implique donc d'admettre (implicitement) que chacun possède les capacités nécessaires à l'exercice politique, c'est-à-dire pour l'essentiel à choisir ceux qui paraissent les plus aptes aux fonctions de commandement.

La démocratie assume ainsi le paradoxe de considérer que c'est « la foule

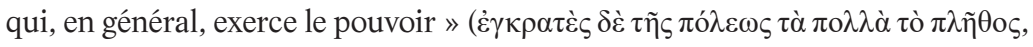
$238 \mathrm{~d} 3-4)$, tout en admettant de déléguer plusieurs fonctions manifestement réfractaires à la gestion collective. Le paradoxe est encore renforcé du fait que, d'un côté, elle revendique un postulat fondamental d'égalité entre tous les citoyens et, de l'autre, elle en juge certains plus aptes au gouvernement. Ce qui soulève la question de l'homogénéité : comment assurer la cohérence du corps des citoyens? Sortir du paradoxe implique donc de clarifier le postulat, ce que fait Aspasie. L'homogénéité démocratique suppose de reconnaître une

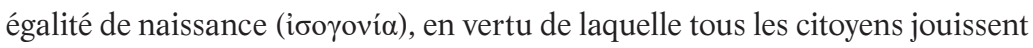
en droit d'un accès identique aux fonctions et aux droits politiques (iøovouía),

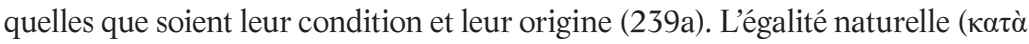

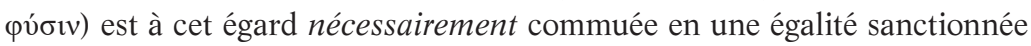

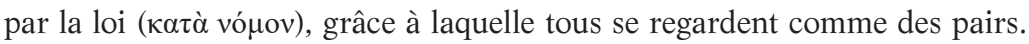
Insister sur l'isonomie montre que l'homogénéité de la démocratie repose moins sur l’idée de peuple $(\delta \tilde{\eta} \mu \circ)$, conçu comme une entité unifiée, que sur

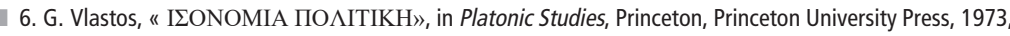
p. 190-192. Rappelons que l'éloge est attribué à une femme, étrangère de surcroît, soit une personne privée de moindre droit politique à Athènes.

7. L'éloge se poursuit jusqu'en 239a5. II doit être comparé à celui que Thucydide attribue à Périclès (II, 37, 1).

8. Sur l'usage dans les epitaphioi logoi de présenter la démocratie comme une aristocratie, je me permets de renvoyer à N. Loraux, L'Invention d'Athènes. Histoire de l'oraison funèbre dans la Cité classique, Paris, Mouton, 1981, chap. IV. 
l'égalité de droit entre ses membres. Aussi la démocratie se caractérise-t-elle essentiellement par l'octroi d'un droit égal à tout citoyen de participer à la vie politique, à la différence de régimes caractérisés par l'inégalité et dépourvus de toute homogénéité, où les uns apparaissent comme des maîtres, les autres comme des esclaves ${ }^{9}$.

Il n'en reste pas moins que certains pouvoirs sont délégués et que certains individus sont jugés meilleurs par leurs pairs (238d). En réalité, la contradiction avec le principe d'égalité est seulement apparente. L'égalité de droit n'oblitère pas les différences entre les individus. Rien n'empêche des citoyens de mieux développer des compétences politiques. La question devient alors celle du critère d'évaluation : qui jugera de la supériorité de certains? Sur ce point, la réponse d'Aspasie est démocratique à deux niveaux. D’une part, c'est bien la foule $(\pi \lambda \tilde{\eta} \theta$ os) qui conserve le pouvoir mais qui prend la décision de le déléguer en partie : les gouvernants intronisés restent les légataires de la cité, à laquelle ils doivent rendre des comptes. D’autre part, leur supériorité est établie par la foule : c'est elle qui juge l'excellence des magistrats à qui elle confie des tâches, sur la base de critères qui lui sont propres, sans jamais prétendre à ce que cette supériorité apparente trouve un quelconque ancrage naturel, réel. En conclusion, la démocratie de droit telle que la présente Aspasie s'accomplit dans une sorte d'aristocratie de fait, qui demeure démocratique dans ses fondements.

\section{Un régime bariolé}

Ce tableau extrêmement favorable prend l'exact contre-pied des critiques de Platon qui, dans la République, attribue à la démocratie les caractéristiques suivantes $^{10}$ : les magistratures y sont tirées au sort; il y règne un excès de liberté ( $\dot{\varepsilon} \lambda \varepsilon v \theta \varepsilon \rho i ́ \alpha)$, de franc-parler ( $\pi \alpha \rho \rho \eta \sigma i ́ \alpha)$ et de licence ( $\dot{\varepsilon} \xi o v \sigma i ́ \alpha)$; s’y retrouvent toutes les autres formes de constitutions; les citoyens n'y subissent aucune contrainte, ni à obéir ni à être obéis, ni à gouverner ni à être gouvernés. Ce résumé le mène naturellement à conclure que la démocratie est, « apparemment, un délicieux régime politique, sans vraie direction, et multicolore, distribuant une certaine forme d'égalité, de façon identique, à ceux qui sont égaux et à ceux qui ne le sont pas ${ }^{11}$. Toute la critique se concentre sur le postulat d'égalité, auquel elle en associe étroitement un autre, plus vigoureux encore en démocratie : le principe de liberté.

\section{Le règne du tirage au sort}

Commençons par l'égalité et par sa première manifestation : le tirage au sort. Si le hasard peut devenir un principe démocratique, c'est grâce au postulat d'une égalité stricte entre les individus, en vertu duquel tout citoyen peut par nature - c'est-à-dire par sa naissance en tant que citoyen - recevoir

9. Ménexène, 238e. De ce point de vue, cette apologie de la démocratie rejoint celle prononcée par Otanès dans le débat sur les constitutions consigné par Hérodote (III, 80).

10. République, VIII, 557a-558a. C'est sans doute ce qui fait dire à Malcolm Schofield que, dans le Ménéxène, Platon se fait « engagé » (Plato. Political Philosophy, op. cit., p. 74), au sens où il s'attaque directement à cette mise à scène glorifiante que la démocratie propose d'elle-même.

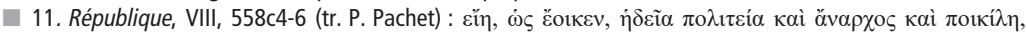

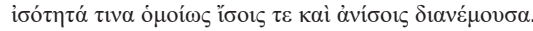


une magistrature, sans que cela ne contrevienne au bien-être général de la cité. Le tirage au sort est en quelque sorte l'accomplissement de l'égalité démocratique, au sens où il implique de négliger complètement les différences de statut ou de capacité à diriger, au motif que chacun possède un droit absolument égal à exercer une fonction politique. Il est remarquable que le discours d'Aspasie passe totalement sous silence cette pratique pourtant caractéristique de la démocratie pour insister sur la valeur aristocratique du régime athénien ${ }^{12}$, ce qui conduit au dilemme suivant. Soit, dans la perspective qui paraît privilégiée par Aspasie, la démocratie délaisse le tirage au sort au profit d'une attribution des magistratures par la voie des élections

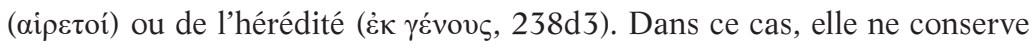
de la démocratie que le nom (et Aspasie note justement que « on l'appelle tantôt démocratie »), vu qu'elle refuse de mener le principe d'égalité jusqu'à sa conséquence ultime. Elle correspond alors à une aristocratie - voire à une oligarchie - de fait, dès lors qu'elle restreint l'accès aux magistratures à ceux qui paraissent les meilleurs et réserve le pouvoir à une élite, restaurant en fait ce qu'elle évacue en droit ${ }^{13}$. Soit, à l'inverse, la démocratie généralise le tirage au sort au nom du principe d'égalité et, du même coup, s'interdit de tirer le meilleur parti de ses citoyens les plus compétents, sinon par le fait $\mathrm{du}$ hasard, dans la mesure où elle refuse d'observer des différences de valeur au moment de déléguer les charges politiques. Le tirage au sort conduit ainsi inévitablement à un nivellement (par le bas) des compétences (et donc de l'exercice) politiques - du moins est-ce là le sens de la critique de Platon.

Entendons-nous. Platon ne conteste ni le tirage au sort ni le principe d'égalité. Il en critique uniquement l'acception démocratique. Ainsi quand, dans les Lois, il examine les sept types de prétention à l'autorité, il place le tirage au sort au sommet de l'échelle, au plus près des dieux parce qu'il résulte d'un choix divin ${ }^{14}$. Il l'utilise ensuite pour diverses procédures d'attribution de charges distinctives au sein de sa cité ${ }^{15}$. Le tirage au sort joue donc un rôle essentiel dans la cité modèle, mais dans une configuration impliquant une sélection, préalable ou a posteriori, qui retient uniquement les citoyens jugés dignes d'exercer la charge en question, en vertu de quoi les meilleurs individus (par la vertu ou par le savoir) accèdent au pouvoir en proportion de leur supériorité. Cette version du tirage au sort s'écarte très manifestement

12. En dépit de son absence dans le panégyrique du Ménéxène, le tirage au sort n'en est pas moins un caractère essentiel de la démocratie grecque, comme cela ressort aussi du passage d'Hérodote mentionné plus haut.

13. Platon insiste par ailleurs sur le risque de dérive qu'encourt la démocratie, non seulement vers l'oligarchie, mais même vers la tyrannie (de fait ou réelle). Dans le Gorgias, il associe ainsi l'orateur et le tyran (466c-d; 467a; 468d; 479a). Dans la République, il fait naître la tyrannie de la démocratie (VIII, 564c-565d).

14. Lois, III, 690c : les autres formes d'autorité qui sont jugées légitimes sont l'autorité parentale, la noblesse, l'ancienneté, la position de maître, la force et le savoir. Ce type de tirage au sort s'avère tout sauf démocratique, puisqu'il concerne les naturels des individus : sont amenés à exercer une autorité légitime ceux qui en possèdent par nature (par le sort) les capacités qui les rendent aptes à diriger.

15. Lois, VI, 756e, 759b et 763d-e. Par exemple, pour le Conseil de la cité pour laquelle il légifère (756c-e), il élabore un système à deux tours. Au premier, les citoyens répartis en quatre classes censitaires votent partout où ils peuvent acquitter le cens (les plus riches votent donc dans les quatre groupes). Au second, tous votent parmi les représentants élus au premier. Ensuite, un tirage au sort ramène les représentants à cent quatre-vingts par classe, qui sont alors soumis à l'examen. Selon Platon, ce procédé concilie monarchie et démocratie, (s)élections et tirage au sort, un juste milieu où doit se tenir la constitution : non seulement les " meilleurs " possèdent plus de poids dans le vote, mais les résultats du tirage au sort sont ensuite passés au crible de la valeur. 
de celle fondée sur l'égalité démocratique, au prétexte que placer l'homme de bien dans une égalité stricte, prétendument naturelle, avec l'homme de peu revient à convertir une inégalité de nature en une égalité apparente, ce que Platon juge profondément inique. À l'égalité arithmétique qui fonde la démocratie, il préfère dès lors l'égalité géométrique, qui accorde un poids politique en proportion de la valeur d'un élément ${ }^{16}$. Au fond, la démocratie ne commet-elle pas l'erreur de postuler une égalité de nature que rien ne vérifie empiriquement? Du moins le dilemme demeure : soit postuler une égalité de principe indiscutable, au détriment de la valeur du gouvernement; soit accommoder l'égalité en faveur d'une méritocratie (dans le meilleur des cas). Nos démocraties représentatives semblent avoir de facto fait leur choix.

\section{Le règne de la liberté}

Si l'égalité s'avère une condition nécessaire de la démocratie, elle ne paraît pas suffisante. Son critère véritable, selon Platon, c'est la liberté. C'est elle en effet que la démocratie pose comme son bien le plus élevé et le plus beau, celui qui la rend digne d'intérêt aux yeux de ses partisans ${ }^{17}$, dans un contexte grec où l'opposition entre servitude et liberté est plus prégnante que celle entre égalité et inégalité. Ce faisant, l'idéal démocratique ne commet aucune erreur de principe. Du moins s'accorde-t-il sur ce point avec le système utopique de la République, qui repose sur la capacité de certains individus à agir et à penser en toute liberté, c'est-à-dire à être capables de se débarrasser des entraves à la décision qui entraîne l'action juste. L'égalité qu'il postule entre les sexes se révèle ainsi une conséquence nécessaire de cet idéal de liberté, dans la mesure où cette capacité appartient à l'un et l'autre sexes. De ce point de vue, l'égalité devient un critère secondaire : les individus dotés de capacités semblables (de commercer, de protéger la cité ou de penser) reçoivent un traitement et des attributions égales, qui dépendent en définitive de leur capacité à manifester la liberté véritable - celle de la pensée. Platon et le courant démocratique s'accordent donc sur la valeur (politique) de la liberté. Ils divergent en revanche sur son extension et sur sa signification.

En démocratie, la liberté procède d'un postulat : elle est simplement reconnue à tous, tout comme l'égalité. Il ne faut ni l'acquérir ni la conquérir, car elle est donnée par nature ou, plus exactement, par la cité à tous ses citoyens. C'est pour cette raison qu'elle est non seulement le bien le plus précieux, celui cultivé pour lui-même, mais aussi le plus fondamental, parce qu'il conditionne tous les autres. Mieux encore, la liberté démocratique se définit comme la licence de "faire ce que l'on veut ${ }^{18}$. Elle est un droit inaliénable qui offre à l'individu la faculté d'agir comme il l'entend, sans que la moindre contrainte (politique) ne pèse sur lui.

16. Voir Lois, VI, 757b-c. Cette égalité proportionnelle séduira Aristote, qui la défendra dans ses Politiques, notamment pour éviter les séditions (III, 16, 2, 1287a12-14; V, 1, 1301a25-39; voir aussi III, 13, 13).

17. République, VIII, 562b-c.

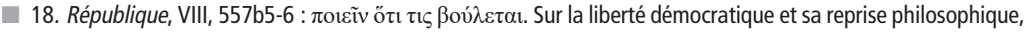
je renvoie à l'article de Bernard Collette-Dučić, « La licence démocratique et son interprétation philosophique dans l'antiquité », dans B. Collette, M.-A. Gavray et J.-M. Narbonne (dir.), Critique et licence dans la Grèce antique, Paris, Les Belles Lettres, 2019, p. 417-442. 
À suivre Platon, le principal effet de cette vision de la liberté est l'individualisme, qui se manifeste à différents niveaux. Le citoyen ne se sent pas tenu de participer à la vie de la cité et son implication politique dépend uniquement de son bon vouloir : il n'éprouve aucunement le besoin d'obéir ou de commander, de mener la guerre aux côtés de ses pairs ou de préserver la paix, de respecter les décisions judiciaires ou de les faire respecter ${ }^{19}$. Même les condamnés se permettent de continuer à circuler librement. La liberté démocratique agit ainsi comme une force centrifuge qui disloque la cité, car elle empêche les citoyens de se sentir impliqués dans un projet commun. Le règne $\mathrm{du}$ « chacun comme il le veut » conduit à celui du « chacun pour soi ». Pour contrer cette tendance et inciter les citoyens à s’investir dans la cité autrement que par l'exercice de leur liberté, la démocratie doit donc les doter de balises grâce auxquelles ils percevront l'importance d'appliquer (et de faire appliquer) les lois, parce qu'ils comprendront que leur liberté, tout inconditionnelle qu'elle est, ne dépend pas moins de la préservation de la cité. Et c'est le rôle que doit remplir l'éducation. Or, en démocratie, l'éducation suit le même modèle libertaire, au sens où chacun est éduqué dans l'idée que la liberté constitue son bien le plus précieux, ce qui se traduit dans sa forme même : il n'y a aucun cadre commun et chacun étudie en définitive les matières qu'il veut ${ }^{20}$. Érigée en principe plutôt qu'en finalité, la liberté entraîne la démocratie vers sa propre dispersion.

Le fait se remarque notamment dans le rapport à l'autorité. Dans tous les autres régimes, le nom que se donnent respectivement les gouvernants et les gouvernés évoque une relation de servitude : les gouvernés se désignent eux-mêmes comme peuple $\left(\delta \tilde{\eta} \mu \varsigma_{\zeta}\right)$ et appellent maîtres $(\delta \varepsilon \sigma \pi$ ó $\tau \alpha$ ı) leurs gouvernants, tandis que ces derniers se qualifient entre eux de codirigeants

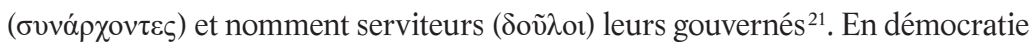
en revanche, l'opposition entre peuple $\left(\delta \tilde{\eta} \mu \varsigma_{\zeta}\right)$ et gouvernants ( $\alpha \rho \chi 0 \nu \tau \varepsilon \varsigma$ ) prévaut. Quelle que soit sa place au sein de l'organisation politique, chacun jouit de la même liberté, et occuper une fonction ne confère aucun droit supplémentaire par rapport à ses concitoyens. Sans doute est-ce pour cette raison que le citoyen d'une démocratie ne ressent aucune attirance à exercer le pouvoir : si sa propre liberté constitue sa seule préoccupation, il acceptera une magistrature si et seulement si il y voit un autre moyen de l'assouvir ${ }^{22}$. Dans tous les autres cas, il préférera s'en abstenir pour continuer à jouir de sa liberté sans entrave. Le parallèle avec le philosophe tel que Platon le décrit est tentant. Si celui-ci est le seul à savoir comment diriger la cité, il estime avoir mieux à faire : sans l'intervention d'une contrainte extérieure, il n'ira jamais en politique de son gré23. Le philosophe platonicien et le citoyen démocratique sont donc animés d'un même désintérêt du pouvoir (pour le pouvoir), au nom d'une aspiration qu’ils jugent supérieure. Toutefois, s’il

21. République, IV, 463a-b. Dans la cité de Platon, les membres du peuple sont appelés donneurs de salaires

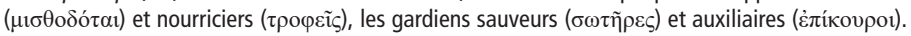

22. Une critique très proche se retrouve chez le Pseudo-Xénophon, Constitution des Athéniens, I, 8.

23. Cf. République, VII, 516c-517a, 519b-d et 520a-d. 
leur arrive jamais d'être contraints à exercer le pouvoir, ils se distingueront dans leur rapport à la liberté. Le premier usera de sa liberté de pensée pour remplir sa tâche : affranchi de toute ambition personnelle grâce à sa science des besoins de la cité et du moyen de les satisfaire, il œuvrera avant tout dans l'intérêt commun, conscient que son propre bien passe par celui de la cité. Le citoyen démocratique, pour sa part, poussé par son seul désir de liberté, subordonnera le bien commun à son intérêt propre. Sans nécessairement donner foi à la thèse de Platon, le contraste invite à s'interroger sur la manière dont la démocratie produit chez ses gouvernants une prise en considération de l'intérêt collectif, dans l'idée d'assortir ce droit à la liberté d'un devoir à son égard.

Mais la question se pose aussi pour les gouvernés, qui se sentent non moins libres que les gouvernants. L'effet conjugué de la liberté et de l'égalité entraîne en effet chez eux une certaine défiance à l'égard de toute forme d'autorité. Que les gouvernants soient tirés au sort parmi tous les citoyens, indistinctement, afin d'exercer une fonction politique sans la moindre

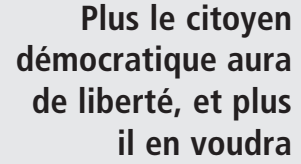
prétention à le faire, implique qu'ils soient sujets à la contestation : pourquoi devrais-je renoncer à ma liberté pour obéir à un individu qui est absolument mon égal et n'a pas plus de légitimité à définir le bien commun, si ce n'est l'effet du sort? Mais, en définitive, le tirage au sort n'est pas le seul motif de réticence et l'élection n'y change rien. De façon générale, en démocratie personne ne se sent tenu par les décisions des dirigeants, car obéir implique non seulement d'accorder une légitimité à la fonction, donc un crédit à ceux qui l'occupent, mais surtout de renoncer à une part de sa liberté individuelle pour suivre les décisions d'un autre. Or pourquoi devrais-je sacrifier ma liberté au profit de la sienne, si nous disposons tous deux d'un droit égal à la liberté? D’après Platon, une telle défiance explique que seuls soient jugés légitimes les dirigeants qui évitent de toucher à la liberté de leurs concitoyens, tandis que les citoyens qui se soumettent délibérément à l'autorité des magistrats optent, aux yeux de leurs semblables, pour la servitude volontaire ${ }^{24}$. Dans ces conditions, la démocratie soulève un vrai problème de rapport à l'autorité et, par extension, d'efficacité réelle du pouvoir : l'institution des magistratures et des fonctions contrevient à ses principes fondamentaux que sont la liberté et l'égalité.

Loin d'être insurmontable, le dilemme peut se résoudre aisément dans le cadre d'un contrat social, en vertu duquel chacun renonce à une part de sa liberté pour la déléguer à un pouvoir commun. Toutefois, le caractère totalement indéterminé de la liberté démocratique, négativité totale, renferme nécessairement une dimension excessive, incontrôlée, illimitée, qui empêche un tel renoncement ${ }^{25}$. En démocratie, même les esclaves et les animaux

24. République, VIII, 562d-e.

25. Plusieurs sophistes ont d'ailleurs été les premiers partisans du contrat social (voir Ch. Khan, "The Origins of Social Contract Theory in the Fifth Century B.C. ", in G.B. Kerferd (ed.), The Sophists and their Legacy, Wiesbaden, Steiner, 1981, p. 92-108; et M. Narcy, "Le Contrat social : d'un mythe moderne à l'ancienne 
d'ordinaire serviles se conduisent de façon impudente et libre, parce qu'il

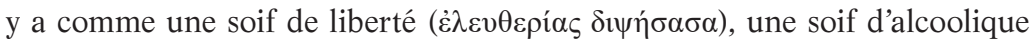
inextinguible et inassouvie ${ }^{26}$. Plus le citoyen démocratique aura de liberté, et plus il en voudra. Plus il en voudra, plus il sera susceptible de s'en prendre aux dirigeants qui contrarieront sa soif, en accusant d'oligarchie ceux qui refusent de le servir.

Cette soif de liberté trouve sans doute sa source dans le principe même de la démocratie. À lire l'exposé de la dégradation progressive des régimes dans la République, la démocratie naît de l'oligarchie, quand les pauvres, lassés d'assister au spectacle de riches dont l'obsession est de s'enrichir, surmontent leur lâcheté pour se débarrasser de ceux-ci et se répartir le pouvoir à égalité ${ }^{27}$. La fiction généalogique ne doit sans doute pas être prise au pied de la lettre. Si elle fait de la démocratie le régime des pauvres, c'est moins pour signifier qu'elle est uniquement peuplée de miséreux que pour insister sur le changement de lieu du désir qui, en passant de l'oligarchie à la démocratie, se déplace de la richesse vers la liberté. En démocratie, la liberté est en quelque sorte le seul bien commun à l'ensemble des citoyens (et des dirigeants). Aussi s'agit-il d'un régime fondé sur la crainte de se retrouver asservi (à nouveau par les riches) et de perdre son seul bien.

Affirmer la liberté sans autre critère que négatif comme le fait la démocratie, se complaire dans une forme de liberté pure (ők $\rho \alpha \tau$ s, 562d2), non mélangée à la moindre contrainte, c'est nécessairement rendre tout exercice du pouvoir impossible. Pour persister, la démocratie doit donc renoncer à une part de sa double caractéristique fondamentale, sans quoi l'unité qu'elle revendique reste de pure façade.

\section{L'homme démocratique, parangon de l'individualisme}

Nous annoncions un second effet de l'égalité démocratique : sa bigarrure. La démocratie procède en effet à une mise à égalité de tous les régimes, qui en fait le régime le plus plaisant, parce qu'elle ressemble à un «bazar aux constitutions $»^{28}$. Cette mise à égalité ne signifie pas que la constitution démocratique emprunte ses composantes à toutes les autres constitutions, au sens où elle contiendrait des éléments tyranniques, oligarchiques, etc. Elle désigne plutôt le fait que la démocratie implique une mise à égalité des individus et des naturels, ainsi que des décisions prises. D’une part, chacun y est libre de vivre comme il le souhaite, c'est-à-dire en suivant les règles qui lui plaisent. Le personnage d'Aristophane, Dicéopolis, le protagoniste des Acharniens, vient directement à l'esprit, lui qui décide de conclure pour lui-même et pour sa famille une trêve avec l'ennemi lacédémonien, plutôt que de continuer à subir les affres de la guerre aux côtés de ses concitoyens. D'autre part, dans les assemblées politiques, chacun prend des décisions

sophistique ", Philosophie 28, 1990, p. 33-56). Toutefois, aucun sophiste n'a fondé explicitement le contrat social sur le renoncement à la liberté, même s'il est vraisemblable qu'ils aient fait de ce renoncement un effet du contrat.

26. République, VIII, 563b-d. La métaphore du vin survient en 562c. Sur ce passage, lire les analyses, brèves mais stimulantes, de R. Muller, La doctrine platonicienne de la liberté, Paris, Vrin, 1997, p. 75-78.

27. Ibid., VIII, 556c-557a.

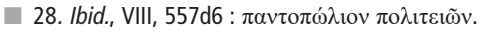


ou, du moins, soutient des motions, résultant de ses propres aspirations. La démocratie a en effet pour caractéristique d'admettre à égalité toutes les décisions ou tous les avis. Sur ce point, le parallèle avec nos assemblées modernes est séduisant, où siègent des partis régis par des visions politiques pour le moins bigarrées, entre les tenants de l'oligarchie, les partisans du retour à un régime autoritaire, les défenseurs d'une dictature du prolétariat... En un sens, loin de constituer un véritable régime politique, qui serait

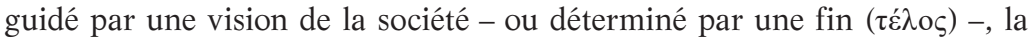
démocratie s'apparente plutôt à une absence de régime défini. Elle est moins une conception de la politique qu'un refus de se prononcer définitivement en faveur d'une vision déterminée de la cité car, de l'avis de Platon, toutes les idées politiques doivent pouvoir s'y exprimer sans entrave, en vertu du principe de liberté qui n'en autorise aucune à monopoliser l'espace public.

Une telle mise à égalité se retrouve au niveau du désir. Dans sa généalogie, Platon apparie en effet chaque constitution à un type de naturel. Sans chercher à dire qu'un régime donné est principalement composé des individus correspondants, il met plutôt en parallèle constitutions et comportements ${ }^{29}$. Pour saisir le naturel démocratique, Platon opère une distinction entre les désirs nécessaires, qui concernent les besoins naturels (se nourrir, etc.), et les désirs superflus, qui vont au-delà ${ }^{30}$. Le naturel démocratique est celui chez qui les seconds prennent le pas sur les premiers, car il met tous les désirs sur un pied d'égalité ( est celui qui réalise indistinctement tous ses désirs, qu'ils contribuent ou non à son bien. Il livre dès lors son âme au commandement du premier désir venu : c'est celui-là qu'il poursuit, car il se trouve pour un temps placé au centre de son attention. Sa vie prend ainsi la forme d'un tirage au sort, où les désirs se succèdent à la faveur du hasard, sans la moindre organisation. Dans ces termes, l'égalité s'avère un refus de hiérarchiser les désirs, donc les vices et les vertus qui les régissent, dans la mesure où tous semblent de même nature et, de ce fait, aucun ne doit être réprimé ${ }^{31}$.

La liberté de l'homme démocratique repose sur un principe d'égalité dont la conséquence ultime s'avère de nier la possibilité même de la liberté en tant que liberté délibérée et résultat d'un projet réfléchi. Parce qu’il y a mise à égalité d'éléments de niveaux différents, le hasard de leur succession devient le seul critère de décision. Or le sort se trouve à l'opposé d'une cause volontaire, rationnelle et libre, bref d'un véritable projet (individuel ou politique), c'est-à-dire d'un projet organisé autour d'un télos. La liberté sans contrainte et sans hiérarchie de la démocratie aboutit en définitive à sa propre négation.

\footnotetext{
29. Dans la République, I'adjectif $\delta \eta \mu \kappa \rho \alpha \tau$ «ó qualifie uniquement I'homme démocratique. II ne reçoit d'ailleurs de sens politique que dans un seul passage des Lois, où il renvoie moins à une démocratie effective qu'à la dimension démocratique d'une constitution (VI, 756 10, cf. supra). Pour désigner la cité démocratique,

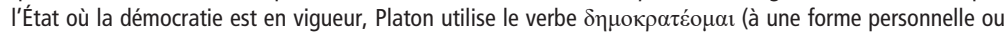
au participe). Quant au substantif $\delta \eta \mu \kappa \rho \alpha \tau i ́ \alpha$, il qualifie un certain type de régime, jamais une cité dont le régime effectif serait une démocratie. Le lexique platonicien observe donc une certaine constance, séparant clairement l'aspect politique de l'aspect psychologique et, au sein de la politique, le type et sa manifestation.

30. Ibid., VIII, 558c-562a.

31. Le portrait du naturel démocratique est sans doute l'un des plus cinglants que Platon ait dressés (561c-e).
} 


\section{Quelle connaissance pour la démocratie?}

Un second horizon de la critique platonicienne de la démocratie est sans doute Protagoras, le premier philosophe à avoir défendu l'idéal démocratique. Platon a dégagé de sa pensée une vision cohérente sur la démocratie, qui forme un second plaidoyer dans les dialogues. Celui-ci explore la transmission des idées démocratiques et le rôle qu'y joue la connaissance. L'enjeu paraît crucial : dans un régime politique où chaque citoyen est amené à participer au pouvoir, comment s'assurer de sa capacité, sinon à considérer que la démocratie ne repose sur aucune compétence spécifique?

\section{Le partage des valeurs}

Si Platon ne met jamais le mot « démocratie » dans la bouche du sophiste, il le contraint à justifier le régime athénien et, du même coup, sa prétention à enseigner l'excellence politique. Protagoras revendique en effet de former de bons citoyens, dans une démocratie grecque qui requiert moins une capacité à exercer des magistratures qu'à participer à la vie de la cité, par une bonne gestion de son patrimoine, par la prise de parole à l'assemblée et par l'exercice d'éventuelles fonctions ${ }^{32}$. C'est dire combien son bon fonctionnement passe par l'éducation. Or Platon formule deux objections ${ }^{33}$. En premier lieu, pour les matières techniques, les Athéniens n'écoutent que les experts, tandis que, pour les questions de politique générale, ils concèdent à chacun un droit de parole égal : par leur attitude, ils rendent donc vain l'enseignement de la politique, vu qu'ils semblent postuler que tous la possèdent en droit. En second lieu, même les leaders démocratiques se révèlent incapables de transmettre leurs compétences à leurs propres fils, et Platon cite Périclès en exemple. Le fonctionnement de la démocratie contredit l'idée d'éducation politique et ressemble à un régime où le pouvoir appartient au premier venu.

Protagoras répond par un mythe qui décompose la répartition des facultés et révèle ainsi leur importance relative pour la vie politique ${ }^{34}$. La justification de la démocratie passe donc à nouveau par la fiction. Négligé par Épiméthée, l'homme est d'abord nu et sans défense. Puis il reçoit de Prométhée le feu et les arts, grâce auxquels il fabrique un langage et des objets techniques utiles à sa subsistance. Enfin, incapable de cohabiter avec ses semblables car l'art politique lui fait encore défaut, il obtient de Zeus la justice ( honte $(\alpha i \delta \omega \varsigma)$, qui sont cette fois distribuées à tout un chacun ${ }^{35}$. En d'autres termes, c'est la participation universelle à cette double capacité politique qui rend la démocratie possible, là où la distribution sélective des savoirs techniques crée certes une relation de dépendance, mais entraîne surtout des torts réciproques ${ }^{36}$. Ce n'est pas parce que je dépends du cordonnier pour me chausser que celui-ci va consentir à me fabriquer des chaussures et moi

32. Platon, Protagoras, désormais "Prot. ", 318e-319a.

33. Ibid., 319b-320b.

34. Ibid., 320c-322d.

35. Pour une analyse approfondie du mythe et des questions connexes, je me permets de renvoyer à mon livre Platon héritier de Protagoras. Dialogue sur les fondements de la démocratie, Paris, Vrin, 2017.

36. Prot., $324 \mathrm{e}-325 \mathrm{a}$. 
à le payer en retour. Il faut encore que nous éprouvions le sens de la justice et de la honte pour être tenus par nos obligations réciproques.

La démocratie repose bien sur un mythe, une croyance partagée et délibérément admise, en vertu duquel chacun possède les capacités indispensables pour prendre part à la politique. Elle fait ainsi l'hypothèse de considérer chacun comme s’il en était doté et refuse que quiconque en soit exclu a priori. Ce postulat rend d'ailleurs la démocratie non seulement possible, mais même nécessaire, étant donné que rien ne justifie d'écarter certains de la vie politique, si tout le monde en détient les capacités. De ce point de vue, simuler la justice, c'est déjà y participer d'une certaine façon, dans la mesure où cela revient à admettre le lien de la cité et à consentir à le préserver $^{37}$. Pour en revenir aux objections de Platon, si la démocratie autorise seulement les spécialistes à se prononcer sur les matières techniques, c'est parce qu'ils sont les seuls compétents dans ces domaines; et si elle autorise tout le monde à s'exprimer sur les questions générales, c'est en vertu de la participation universelle aux capacités politiques. Qu'en est-il à présent de la transmission des compétences politiques? La démocratie n'est-elle rien d'autre qu’un régime de l’opinion immédiate et du talent inné? Sans être encore des vertus, mœurs ou règles instituées, justice et honte sont des capacités à transformer en compétences. Il reste à expliquer comment les développer pour acquérir la connaissance des normes de la cité.

L'éducation démocratique constitue un processus marqué par des ajustements constants, ce que Protagoras explique au moyen d'une analogie avec la langue maternelle : l'apprentissage ne repose pas sur un seul professeur clairement identifiable, mais tous l'enseignent à tous, en permanence et dès l'enfance ${ }^{38}$. Du fait que chacun a les capacités nécessaires, il peut recevoir d'autrui un savoir avant de le/lui transmettre (à) un autre. Chacun occupe tour à tour la place de maître et de disciple, devenant progressivement spécialiste dans les matières politiques ${ }^{39}$. La démocratie suppose donc que chacun accepte de prendre part en permanence à l'éducation politique. L'analogie linguistique permet d'aller plus loin : les citoyens contribuent aux lois comme à l'évolution de la langue, de façon impersonnelle, collective et continue. Ils s'avèrent à cet égard responsables de leur propre éducation et de leur propre unité politique. Maillons indispensables de la démocratie, ils s'intègrent au mouvement qui les englobe et les dépasse, qu'ils contribuent à entretenir. L'universalité des facultés politiques conduit en quelque sorte à instituer une technocratie où l'expertise est l'apanage de chacun, permettant la construction collective d'un projet commun, d'une unité qui se modifie et s'adapte sans cesse.

Ce processus n'empêche pas cependant l'émergence d'inégalités. Si Protagoras croit en la force de l'éducation, les faits le conduisent à reconnaître une hiérarchie des compétences, car certains se montrent plus doués que d'autres. Or l'écart reste ténu, une différence minime de degré (ỏ í́yov, 328a) qui ne justifie pas d'instituer une aristocratie. Les meilleurs sont plutôt une 
chance pour la communauté, au sens où ils contribueront à élever d'un degré l'éducation. En d'autres termes, tout en admettant l'égalité de principe des capacités politiques, Protagoras évite de conclure que chacun les réalise au même degré. La démocratie doit postuler l'égalité, mais pas nécessairement admettre que tout le monde se vaut quant aux compétences acquises. Surviennent bien des inégalités, mais qui seront l'occasion d'une amélioration collective.

En conclusion, l'apologie de la démocratie que prononce Protagoras propose une vision alternative du postulat d'égalité, qui ne se fonde plus sur la reconnaissance de droits, mais en justifie la possibilité. Dans ses termes, la démocratie fait l'hypothèse d'une distribution universelle des capacités politiques, dont elle pose que tout le monde est doté a priori, ce qui l'autorise à reconnaître à tous un droit égal à la vie politique. Pour justifier a posteriori son hypothèse, elle considère l'éducation politique comme un processus continu et réciproque, qui s'étend sur l'ensemble de l'existence, en vertu duquel chacun devient compétent et actualise les capacités politiques qu'il possède en droit.

\section{L'absence de connaissance}

Comme Protagoras, Platon soutient que la politique suppose un savoir. Il s'accorde même à dire que maîtriser une science (ou un art) exige à la fois du temps et une prédisposition de l'individu ${ }^{40}$. Leur divergence concerne la nature de la science politique. Pour Protagoras, elle consiste en trois éléments : l'ensemble des règles de la cité, la manière de les respecter et les moyens de les modifier. Or, du fait que ces règles sont édictées par des citoyens tous pourvus des mêmes capacités politiques, il résulte que leur connaissance et leur mise en œuvre sont, en droit, accessibles à tous. De ce point de vue, le savoir démocratique correspond à un savoir (immanent) du fonctionnement de la cité, que certains possèdent mieux parce qu'ils éprouvent avec plus d'acuité la nature de ses besoins et le moyen de les satisfaire, c'est-à-dire de la faire évoluer vers un état meilleur pour elle ${ }^{41}$.

Platon estime en revanche qu'aucun savoir ne peut être maîtrisé par la totalité des citoyens, ni même par leur majorité, parce que l'apprentissage requiert un temps et une concentration incompatibles avec une activité professionnelle. C'est la thèse de la République, qui exclut de la vie politique toute la population à l'exception de la catégorie des gardiens ${ }^{42}$. C'est aussi la thèse du Politique, où Platon examine les constitutions à l'aune de son modèle, qui repose sur la dialectique appliquée au gouvernement de la cité ${ }^{43}$. Il les envisage ainsi sous le critère du savoir : la seule constitution droite est

40. Platon brosse un tableau qui rejoint les rares fragments de Protagoras sur l'éducation : " II disait que l'art sans la pratique et la pratique sans l'art ne valent rien » (B 10 DK) et " La culture n'émerge pas dans l'âme si on ne la pénètre pas en profondeur " (B 11 DK). Voir aussi "L'enseignement requiert de la nature et de l'exercice » et " Il faut commencer à apprendre dès l'enfance » (B $3 \mathrm{DK})$.

41. Sur ce point, le Protagoras rejoint la définition du savoir que Platon attribue au sophiste dans le Théétète, où il consiste, dans le cas de l'orateur, en une capacité à modifier ce que la cité considère comme juste pour qu'y entrent des contenus plus utiles (Théétète, 167b-d). Je renvoie à nouveau à mon Platon héritier de Protagoras.

42. Ce point a été abondamment étudié, mais je ne résiste pas à renvoyer aux pages qu'y a consacrées J. Rancière, Le philosophe et ses pauvres, Paris, Flammarion, 20072, p. 17-52.

43. Politique, 292a-e. Sur la thèse du Politique, je renvoie au livre récent de D. El Murr, Savoir et gouverner. Essai sur la science politique platonicienne, Paris, Vrin, 2014. 
celle régie par la science politique, grâce à laquelle le politique adapte en permanence ses décisions à la situation. Incapable cependant de vivre au chevet de chaque citoyen, le politique édicte des lois, qui imitent son savoir. La loi est en effet une opinion, un énoncé associé à une situation pour mener la cité dans la bonne direction. Elle offre une solution de repli commode, à défaut d'être en permanence la plus adéquate, car elle présente le mérite de s'appliquer à tous de façon uniforme et évite ainsi les dérives de l'arbitraire. Faute de savoir politique, il est donc dans l'intérêt des constitutions de la respecter. Il arrive néanmoins qu'elles ne suivent ni la loi ni le savoir, et sont alors les pires de toutes : la tyrannie et l'oligarchie. Où la démocratie se situe-t-elle dans cette typologie?

Platon range la démocratie dans les constitutions déviées. Il en fait le « gouvernement de la foule » $(\dot{\eta}$

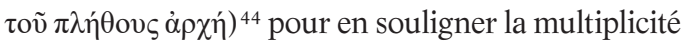
constitutive et évincer l'unité d'une entité identifiable, celle du peuple $(\delta \tilde{\eta} \mu \circ \varsigma)$, à laquelle l'étymologie laisserait croire. La démocratie apparaît comme la constitution où le pouvoir est distribué entre tous les

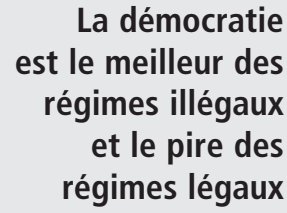
citoyens. Elle implique en ce sens une dispersion des avis et manque du sentiment commun qui la ferait parler d'une seule voix. En même temps qu'il rend son unité problématique, cet éparpillement l'affaiblit résolument, tant pour le meilleur que le pire : une telle dilution du pouvoir entraîne nécessairement de l'inertie, car l'excès dans la formulation d'avis divers et variés empêche la prise de décision, même s'il diminue du même coup les risques de dérive. Régime dévié le plus inertiel, la démocratie paraît être pour la même raison le moins dangereux et le plus doux à vivre ${ }^{45}$. Mais la dissémination du pouvoir enlève également toute importance au respect des lois : le pouvoir étant exercé par tous et sur tous, aucune décision ne peut causer de peines extrêmes à qui que ce soit. Sous cet angle, la démocratie est donc le meilleur des régimes illégaux et le pire des régimes légaux, en raison de l'inefficacité que produit inéluctablement la fragmentation du pouvoir. Le plus tiède des régimes, en quelque sorte.

Mais la fragmentation du pouvoir est également fragmentation du savoir. Son émiettement mène tout droit à un régime de l'opinion et de la répétition : selon Platon, dans un régime où tous enseignent à tous le savoir politique, celui-ci se fonde nécessairement sur le passé, sur les opinions acquises. Il consiste en la reproduction inlassable des mêmes recettes, celles qui ont déjà fait leurs preuves et doivent continuer à fonctionner. La démocratie devient le régime de la reproduction, où se manifeste à nouveau son inertie.

Se pose dès lors la question du mode de savoir et de vérité que la démocratie peut espérer. Platon laisse penser que la démocratie ne peut être le lieu d'une vérité et d'un savoir absolus. Il attribue d'ailleurs à Protagoras une théorie de la vérité qui est adaptation aux circonstances et ajustement constant. Mais 
ce n'est pas sur le point de l'évolution permanente que Platon s'y oppose, lui qui prône un modèle politique d'adaptation au kairos ${ }^{46}$. C'est plutôt dans la nature du savoir que se situe la différence. Pour Platon, l'adaptation aux circonstances n'interdit pas d'atteindre une vérité ni d'être en mesure de déclarer une décision la meilleure possible. Pour la vision démocratique incarnée par Protagoras, le savoir doit en revanche renoncer au régime du vrai pour entrer dans celui du plus et du moins, du meilleur et du pire. Il évacue le principe d'une décision meilleure tout court pour celui d'une décision meilleure qu'une autre. Dans ce cadre, la démocratie est le régime qui renonce au régime du vrai.

Toutefois, ce que dénonce Platon, c'est la tendance en démocratie à maintenir l'aspiration au vrai. Ainsi, quand nous faisons appel à des experts, à des politiciens, nous attendons d'eux qu'ils délivrent la vérité qui conduit à la décision. Or cette vérité sur laquelle nous comptons, Platon ajoute, nous la jugeons selon nos propres critères, qui sont des critères d'ignorance : ce sont en définitive les citoyens qui jugent de la valeur de la vérité qu'ils estiment pourtant être absolue. De plus, ils évaluent les propositions qui leur sont faites en fonction de leurs propres attentes, de leurs propres opinions ${ }^{47}$. Ils vivent ainsi dans l'attente d'une vérité, d'une solution définitive, mais ils la jugent en fonction de leur point de vue ignorant.

Platon estime pour cette raison que la foule n'a pas à juger du travail des politiques - du moins des vrais politiques, ceux qui détiennent la véritable science politique. Protagoras, au contraire, soutient qu'il appartient également au travail du politique d'améliorer la disposition de la cité, c'est-à-dire d'user de la rhétorique pour montrer que le résultat est meilleur. Mais la question de la justification du critère du mieux reste entière. Qui au fond, en démocratie, juge-t-il du mieux? Est-ce le monopole d'expert qui doit imposer son point de vue? Mais au nom de quoi? Est-ce plutôt le privilège de tous les citoyens? Mais comment éviter de se contenter de répondre à leurs attentes? Le mieux pour les individus ne sera peut-être pas le mieux pour la collectivité : chacun s'en trouve peut-être mieux, mais pas l'ensemble. Le problème se pose une nouvelle et dernière fois : comment la démocratie parviendra-t-elle à réconcilier l'intérêt du tout avec celui de chacune de ces parties?

\section{Le rêve démocratique}

Avec les siècles, nous avons intériorisé la définition (aristotélicienne) de l'homme comme un animal politique. Sans plus guère nous soucier du sens original de l'énoncé, nous estimons désormais naturel que tout homme participe à la vie politique et que le meilleur régime, celui qui est le plus adapté à la nature humaine, soit donc la démocratie. Notre aspiration va peut-être même plus loin encore : puisque nous sommes tous citoyens, politiques, ne devrionsnous pas tous également être philosophes, au sens où le citoyen véritable en démocratie serait celui qui exerce sa capacité critique? Si Platon nie la possibilité de cette fiction démocratique, c'est avant tout parce que la démocratie 
s'avère incapable d'enseigner ses propres valeurs, de faire véritablement en sorte que tous ses citoyens exercent réellement leurs compétences politiques, comme elle le prétend. Selon Platon, être un citoyen, être politique au sens propre du terme, n'est pas en effet un droit ou une fonction. Cela exige des compétences effectives. Il ne suffit pas, selon lui, d'attribuer à tout un chacun des facultés politiques de iure. Certes, l'hypothèse a ses vertus et s'avère difficile à contester. Mais le modèle démocratique d'enseignement, tel que le conçoit Protagoras, ne parvient jamais à sortir de l'opinion et d'un régime de répétition. Le défi de la démocratie est donc de parvenir à développer les compétences politiques qu'elle reconnaît par hypothèse à tous ses citoyens, sans exception, de façon à ce que tous deviennent vraiment citoyens et pleinement politiques. Du moins est-ce le défi que Platon nous oblige à relever.

Marc-Antoine Gavray FRS-FNRS / Université de Liège 